\title{
On the long-time behavior of the solution of a non linear viscoelastic plate equation with infinite memory and general kernel
}

\author{
Adel M. Al-Mahdi, \\ The Preparatory Year Program, King Fahd University of Petroleum and Mineralsy, \\ Dhahran 31261, \\ Saudi Arabia.

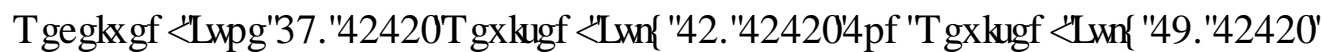

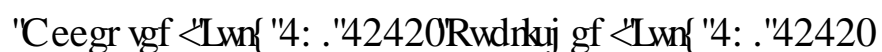

\begin{abstract}
In this paper, we investigate the asymptotic behavior of the solution of a nonlinear viscoelastic plate equation with infinite memory. The nonlinearity in this problem is of a logarithmic type. We use a minimal condition on a relaxation function $h \in L^{1}(0, \infty)$; that is

$$
h^{\prime}(t) \leq-\xi(t) H(h(t))
$$

where $\xi$ is a nonincreasing function and $H$ is an increasing and convex function near the origin. We establish an explicit energy decay formula under this very general assumption on the behavior of the relaxation function at infinity. Our results substantially improve some earlier results in the literature.
\end{abstract}

Keywords- Asymptotic behavior, Convex functions, Infinite memory, Logarithmic Sobolev inequalities, Plate equation.

\section{INTRODUCTION}

Viscoelastic plate equations have been studied by many authors and several stability results have been established. For example, Dafermos 11 considered the following abstract problem with infinite memory

$$
u_{t t}+A u-\int_{0}^{+\infty} h(s) A u(t-s) d s=0, t>0
$$

where $A$ is a strictly positive self-adjoint linear operator and he proved that the energy tends asymptotically to zero, but no decay rate was given. Appleby et al. 2 studied the linear integro-differential equation

$$
u_{t t}+A u(t)+\int_{-\infty}^{t} h(t-s) A u(s) d s=0, t>0,
$$

and established an exponential decay result for strong solutions in a Hilbert space. Pata 3 discussed the decay properties of the semigroup generated by the following equation:

$u_{t t}+\alpha A u(t)+\beta u_{t}(t)-\int_{0}^{+\infty} h(s) A u(t-s) d s=0, t>0$,

where $\alpha>0, \beta \geq 0$ and the memory kernel $h$ is a decreasing function satisfying specific conditions. Subsequently, they established necessary as well as the sufficient conditions for the exponential stability. In [4], Guesmia considered

$$
u_{t t}+A u-\int_{0}^{+\infty} h(s) A u(t-s) d s=0, t>0,
$$

and introduced a new approach for proving a more general decay result based on the properties of convex functions and the use of the generalized Young inequality. He used a larger class of infinite history relaxation functions satisfies the following condition

$$
\int_{0}^{+\infty} \frac{h(s)}{H^{-1}\left(-h^{\prime}(s)\right)} d s+\sup _{s \in \mathbb{R}_{+}} \frac{h(s)}{H^{-1}\left(-h^{\prime}(s)\right)}<+\infty,
$$

such that

$$
H(0)=H^{\prime}(0)=0 \text { and } \lim _{t \rightarrow+\infty} H^{\prime}(t)=+\infty
$$

where $H: \mathbb{R}_{+} \rightarrow \mathbb{R}_{+}$is an increasing strictly convex function. Al-Mahdi and Al-Gharabli 5 considered the following

$u_{t t}-\Delta u+\int_{0}^{+\infty} h(s) \Delta u(t-s) d s+\left|u_{t}\right|^{m-2} u_{t}=0$, in $D$,

where $D=\Omega \times(0,+\infty)$. They established decay results with using a relaxation function $h$, satisfying the following condiation

$$
h^{\prime}(t) \leq-\xi(t) h^{p}(t), t \geq 0,1 \leq p<\frac{3}{2} .
$$


Mustafa 6] consider the following coupled quasilinear system

$$
\left\{\begin{array}{l}
\left|u_{t}\right|^{\rho} u_{t t}-\Delta u-\Delta u_{t t}+\int_{0}^{t} g_{1}(s) \Delta u(t-s) d s+f_{1}(u, v)=0 \\
\left|v_{t}\right|^{\rho} v_{t t}-\Delta v-\Delta v_{t t}+\int_{0}^{\infty} g_{2}(s) \Delta v(t-s) d s+f_{2}(u, v)=0
\end{array}\right.
$$

and established more general decay rate results where the relaxation functions satisfy $g_{i}^{\prime}(t) \leq-H\left(g_{i}(t)\right), i=1,2$. He provided more general decay rates for which the usual exponential and polynomial rates are only special cases. Recently, Al-Mahdi [7] consider the following viscoelastic plate problem with a velocity-dependent material density and a logarithmic nonlinearity:

$\left|u_{t}\right|^{\rho} u_{t t}+\Delta^{2} u+\Delta^{2} u_{t t}-\int_{0}^{+\infty} g(s) \Delta^{2} u(t-s) d s=k u \ln |u|$,

in $D$, where $D=\Omega \times(0, \infty)$ and $\Omega$ is a bounded domain of $\mathbb{R}^{2}$, with a smooth boundary $\partial \Omega$. He established an explicit and general decay rate results with imposing a minimal condition on the relaxation function, that is,

$$
g^{\prime}(t) \leq-\xi(t) H(g(t))
$$

where the two functions $\xi$ and $H$ satisfy some conditions. Very recently, Al-Mahdi 8 considered the following plate problem:

$$
u_{t t}-\sigma \Delta u_{t t}+\Delta^{2} u-\int_{0}^{+\infty} g(s) \Delta^{2} u(t-s) d s=0,
$$

and proved that the stability of this problem holds for which the relaxation function $g$ satisfies the same condition (11). For a numerical study of a viscoelastic flow between porous moving walls, we refer to see 9]. For more result in this direction, we refer the reader to see 10,20$]$.

A. Problems with Logarithmic Nonlinearity and their Applications

The logarithmic nonlinearity has many applications in physics such as nuclear physics, optics and geophysics 2126 . For the problems with logarithmic nonlinearity, we start with the works of Birula and Mycielski 21] and 27] where they proved that the wave equations with the logarithmic nonlinearity have stable and localized solutions. Cazenave and Haraux 28 looked into the following Cauchy problem

$$
u_{t t}-\Delta u=u \ln |u|^{\alpha}
$$

in $\mathbb{R}^{3}$. They established the existence and uniqueness of the solution. The corresponding one-dimensional problem of 12 is studied by Gorka 22 where he establish the global existence of weak solutions provided that $\left(u_{0}, u_{1}\right) \in H_{0}^{1} \times L^{2}$. In 23], Bartkowski and Gorka investigated the weak solutions and also proved existence results of the classical solutions. Hiramatsu et al. 24 considered the following problem

$$
u_{t t}-\Delta u+u+u_{t}+|u|^{2} u=u \ln |u|
$$

and they investigated the numerical solutions of this problem without theoretical analysis. Recently, AlGharabli et al. 25] considered the following

$$
u_{t t}+\Delta^{2} u+u-\int_{0}^{t} h(t-s) \Delta^{2} u(s) d s=\alpha u \ln |u|,
$$

in $\Omega \times(0, \infty)$ and proved the existence and decay results of the solutions with imposing the following condition on the relaxation function

$$
h^{\prime}(t) \leq-\xi(t) h^{p}(t), 1 \leq p<\frac{3}{2},
$$

where $\alpha$ in 14 is a positive constant satisfies $\alpha<\alpha_{0}$ and

$$
\sqrt{\frac{2 \pi \ell}{\alpha_{0} c_{p}}}=e^{-\frac{3}{2}-\frac{1}{\alpha_{0}}}
$$

where $c_{p}$ is a positive constant satisfies the poincare inequality. In [26], Al-Gharabli et al. considered the following problem

$\left|u_{t}\right|^{\rho} u_{t t}+\Delta^{2} u+\Delta^{2} u_{t t}-\int_{0}^{t} h(t-s) \Delta^{2} u(s) d s=\alpha u \ln |u|$,

in $\Omega \times(0, \infty)$ and as in 25 the authors proved the existence and decay results of the solutions with imposing the same two conditions $(15)$ and $(16)$. Very recently, Al-Gharabli 29] considered the same problem (14) with finite memory and established a general decay result for which the relaxation function $h$ satisfies $h^{\prime}(t) \leq-\xi(t) H(h(t))$. For more results of some problems with logarithmic nonlinearity, we refer to the recent works in 3034 .

\section{B. Our Problem and Motivations}

Motivated by these importance and applications of the problems with logarithmic nonlinearity and the the lake of decay results of such those problems with using a wider class of the relaxation functions, we consider the following viscoelastic plate problem with logarithmic nonlinearity:

$\begin{cases}u_{t t}+\Delta^{2} u+u-\int_{0}^{+\infty} h(s) \Delta^{2} u(t-s) d s=\alpha u \ln |u|, & \text { in } D, \\ u=\frac{\partial u}{\partial \nu}=0, & \text { in } \partial \Omega \times(0, \infty), \\ u(x,-t)=u_{0}(x, t), & u_{t}(x, 0)=u_{1}(x), \quad \text { in } \Omega,\end{cases}$

where $D=\Omega \times(0, \infty)$ and $\Omega \subseteq \mathbb{R}^{2}$ is a bounded domain with a smooth boundary $\partial \Omega$. The vector $\nu$ is the unit outer normal to $\partial \Omega$ and the constant $\alpha$ is a small positive real number measures the force of the nonlinear interaction and the nonlinear effects in quantum mechanics. The function $h$ is the kernel and satisfies some conditions to be specified later.

In the present paper, we investigate the stability of the solution of our problem. In fact, we extend some earlier works for wave equations to the plate equation with logarithmic nonlinearity. We also extended some general decay results, known for the case of finite history, to the case of infinite history where the relaxation function satisfies a wider class of relaxation functions. Moreover, we 
drop the boundedness assumptions on the history data considered in many earlier results in the literature.

Our Methodology: We obtained our results by using the multiplier method with some logarithmic inequalities and some properties of integro-differential equations and inequalities. Our decay result is based on $\xi, H$ and $\alpha$.

Existing and Alternative Approaches: The existing approaches in the literature to prove the stability of our problem (17) exist in 25 and [26], however, the relaxation function $h$ is especial case of our relaxation function defined in (19). Moreover, the same relaxation function used in the present paper is used in 7] and [8, however, that approach is completely different. The decay rate obtained in $[7]$ and 8 is better than the decay rate obtained in the present paper but the current approach is much better and easier than the one in 7 and 8 .

This paper is organized as follows. In section (II.), we present some notations, assumptions and the local and global existence result of our problem. In section (III.), we establish some lemmas needed in the proof of our result. The stability results with an example are presented in section (IV.). Some conclusions and future works are mentioned in Section (V.).

\section{Preliminaries}

In this section, we present some notations and material needed in the proof of our results. We use the standard Lebesgue space $L^{2}(\Omega)$ and Sobolev space $H_{0}^{2}(\Omega)$ with their usual scalar products and norms. Throughout this paper, $c$ is used to denote a generic positive constant and we consider the following hypotheses:

(A1) $h: \mathbb{R}^{+} \rightarrow \mathbb{R}^{+}$is a $C^{1}$ - nonincreasing function satisfying, for some $\beta_{0}>0$,

$$
-\beta_{0} h(s) \leq h^{\prime}(s), h(t)>0,1-\int_{0}^{+\infty} h(s) d s:=\ell>0,
$$

(A2) There exists a function $H:(0, \infty) \rightarrow(0, \infty)$ in $C^{1}\left(\mathbb{R}_{+}\right) \cap C^{2}\left(\mathbb{R}_{+}^{*}\right)$ which is increasing and strictly convex, with $H(0)=H^{\prime}(0)=0, \lim _{s \rightarrow+\infty} H^{\prime}(s)=$ $+\infty, s \mapsto s H^{\prime}(s)$ and $s \mapsto s\left(H^{\prime}\right)^{-1}(s)$ are convex on $(0, r]$ and there exists a nonincreasing function $\xi: \mathbb{R}_{+} \rightarrow \mathbb{R}_{+}$, such that

$$
h^{\prime}(t) \leq-\xi(t) H(h(t)), \quad \forall t \geq 0 .
$$

(A3) The constant $\alpha$ in 17 is such that

$$
0<\alpha<\alpha_{0}=\frac{2 \pi \ell e^{3}}{c_{p}},
$$

where $c_{p}$ is the smallest positive number satisfying

$$
\|\nabla u\|_{2}^{2} \leq c_{p}\|\Delta u\|_{2}^{2}, \quad \forall u \in H_{0}^{2}(\Omega)
$$

where $\|\cdot\|_{2}=\|\cdot\|_{L^{2}(\Omega)}$.

Remark 1 Assumption (A3) is needed for establishing the local existence of the solutions of the problem (17). For more details we refer to see [25].
Remark 2 If $H$ is a strictly increasing and strictly convex $C^{2}$ function on $(0, r]$, with $H(0)=H^{\prime}(0)=0$, then it has an extension $\bar{H}$, which is strictly increasing and strictly convex $C^{2}$ function on $(0,+\infty)$. For instance, if $H(r)=a, H^{\prime}(r)=b, H^{\prime \prime}(r)=C$, we can define $\bar{H}$, for $t>r$, by

$$
\bar{H}(t)=\frac{C}{2} t^{2}+(b-C r) t+\left(a+\frac{C}{2} r^{2}-b r\right) .
$$

For simplicity, in the rest of this paper, we use $H$ instead of $\bar{H}$

Remark 3 Since $H$ is strictly convex on $(0, r]$ and $H(0)=0$, then

$$
H(\theta t) \leq \theta H(t), 0 \leq \theta \leq 1 \text { and } t \in(0, r] .
$$

Remark 4 The function $g(s)=\sqrt{\frac{2 \pi \ell}{c_{p} s}}-e^{-\frac{3}{2}-\frac{1}{s}}$ is a continuous and decreasing function on $(0, \infty)$, with

$$
\lim _{s \rightarrow 0^{+}} g(s)=\infty \text { and } \lim _{s \rightarrow \infty} g(s)=-e^{-\frac{3}{2}} .
$$

Then, there exists a unique $\alpha_{0}>0$ such that $g\left(\alpha_{0}\right)=0$. Moreover,

$$
e^{-\frac{3}{2}-\frac{1}{s}}<\sqrt{\frac{2 \pi \ell}{c_{p} s}}, \forall s \in\left(0, \alpha_{0}\right)
$$

which implies that the selection of $\alpha$ in $(A 3)$ is possible.

The modified energy functional associated with problem (17) is given by

$$
\begin{aligned}
E(t) & =\frac{1}{2}\left(\left\|u_{t}\right\|_{2}^{2}+\ell\|\Delta u\|_{2}^{2}+\frac{\alpha+2}{2}\|u\|_{2}^{2}\right) \\
& -\frac{1}{2} \int_{\Omega} u^{2} \ln |u|^{\alpha} d x+\frac{1}{2}(h o \Delta u)(t),
\end{aligned}
$$

where

$$
(h o \Delta u)(t)=\int_{0}^{+\infty} h(s)\|\Delta u(t)-\Delta u(t-s)\|_{2}^{2} d s .
$$

Direct differentiation of (24) with using (17), leads to

$$
E^{\prime}(t)=\frac{1}{2}\left(h^{\prime} o \Delta u\right)(t) \leq 0 .
$$

Lemma 1 [35, 36] (Logarithmic Sobolev inequality) Let $u$ be any function in $H_{0}^{1}(\Omega)$ and $a$ be any positive real number. Then

$\int_{\Omega} u^{2} \ln |u| d x \leq \frac{1}{2}\|u\|_{2}^{2} \ln \|u\|_{2}^{2}+\frac{a^{2}}{2 \pi}\|\nabla u\|_{2}^{2}-(1+\ln a)\|u\|_{2}^{2}$.

Corollary 1 Let $u$ be any function in $H_{0}^{2}(\Omega)$ and a be any positive real number. Then

$\int_{\Omega} u^{2} \ln |u| d x \leq \frac{1}{2}\|u\|_{2}^{2} \ln \|u\|_{2}^{2}+\frac{c_{p} a^{2}}{2 \pi}\|\Delta u\|_{2}^{2}-(1+\ln a)\|u\|_{2}^{2}$. 
Now, we state without proofs the following existence result of the solution of our problem (17).

Theorem 1 Let $\left(u_{0}, u_{1}\right) \in H_{0}^{2}(\Omega) \times L^{2}(\Omega)$. Assume that $(A 1)-(A 3)$ and the following selection of a

$$
e^{-\frac{3}{2}-\frac{1}{\alpha}}<a<\sqrt{\frac{2 \pi \ell}{\alpha c_{p}}}
$$

hold. Then problem (17) has a weak solution

$u \in C\left([0, T], H_{0}^{2}(\Omega)\right) \cap C^{1}\left([0, T], L^{2}(\Omega)\right) \cap C^{2}\left([0, T], H^{-2}(\Omega)\right)$

For the global existence, we introduce the following functionals:

$$
\begin{aligned}
J(t)=\frac{1}{2}\left(\ell\|\Delta u\|_{2}^{2}+\right. & \left.\|u\|_{2}^{2}+(h o \Delta u)(t)-\int_{\Omega} u^{2} \ln |u|^{\alpha} d x\right) \\
& +\frac{\alpha}{4}\|u\|_{2}^{2}
\end{aligned}
$$

and

$$
I(t)=\ell\|\Delta u\|_{2}^{2}+\|u\|_{2}^{2}+(h o \Delta u)(t)-3 \int_{\Omega} u^{2} \ln |u|^{\alpha} d x .
$$

From 30 and 31 , one can easily see that

$$
J(t)=\frac{1}{3}\left[\ell\|\Delta u\|_{2}^{2}+\|u\|_{2}^{2}+(h o \Delta u)(t)\right]+\frac{\alpha}{4}\|u\|_{2}^{2}+\frac{1}{6} I(t) .
$$

Therefor, we have the following important lemma

Lemma 2 Assume that $(A 1)-(A 3)$. Let $\left(u_{0}, u_{1}\right) \in$ $H_{0}^{2}(\Omega) \times L^{2}(\Omega)$ such that

$$
I(0)>0 \text { and } \sqrt{54} k c_{*}^{3}\left(\frac{E(0)}{\ell}\right)^{\frac{1}{2}}<\ell
$$

Then

$$
I(t)>0, \forall t \in[0, T) .
$$

The proofs of the above existence results can be established by following the same arguments in 25] with adapting the finite memory to infinite memory.

\section{TECHNICAL LEMMAS}

In this section, we start by establishing several lemmas needed for the proof of our main result.

Lemma 3 There exists a positive constant $M_{1}$ such that

$$
\int_{t}^{\infty} h(s)(\triangle u(t)-\triangle u(t-s))^{2} d s d x \leq M_{1} h_{1}(t),
$$

where $h_{1}(t):=\int_{0}^{+\infty} h(t+s)\left(1+\left\|\triangle u_{0}(s)\right\|^{2}\right) d s$.
Proof The proof is based on the same arguments in [8]. In fact, we have

$$
\begin{aligned}
& \int_{t}^{+\infty} h(s)\|\triangle u(t)-\triangle u(t-s)\|^{2} d s \\
& \leq 2\|\triangle u(t)\|^{2} \int_{t}^{+\infty} h(s) d s+2 \int_{t}^{+\infty} h(s)\|\triangle u(t-s)\|^{2} d s \\
& \leq 2 \sup _{s \geq 0}\|\triangle u(s)\|^{2} \int_{0}^{+\infty} h(t+s) d s+2 \int_{0}^{+\infty} g(t+s)\|\triangle u(-s)\|^{2} d s \\
& \leq\left(\frac{4}{\ell} E(s)\right) \int_{0}^{\infty} h(t+s) d s+2 \int_{0}^{\infty} h(t+s)\left\|\triangle u_{0}(s)\right\|^{2} d s \\
& \leq\left(\frac{4}{\ell} E(0)\right) \int_{0}^{+\infty} h(t+s) d s+2 \int_{0}^{+\infty} h(t+s)\left\|\triangle u_{0}(s)\right\|^{2} d s \\
& \leq M_{1} \int_{0}^{+\infty} h(t+s)\left(1+\left\|\triangle u_{0}(s)\right\|^{2}\right) d s,
\end{aligned}
$$

where $M_{1}=\max \left\{2, \frac{4 E(0)}{\ell}\right\}$.

Lemma 4 Assume that $h$ satisfies $(A 1)$. Then, for $u \in$ $H_{0}^{2}(\Omega)$,

$$
\begin{aligned}
& \int_{\Omega}\left(\int_{0}^{+\infty} h(s)(\Delta u(t)-\Delta u(t-s)) d s\right)^{2} d x \leq c(h \circ \Delta u)(t), \\
& \int_{\Omega}\left(\int_{0}^{+\infty} h^{\prime}(s)(\Delta u(t)-\Delta u(t-s)) d s\right)^{2} d x \leq-c\left(h^{\prime} \circ \Delta u\right)(t) .
\end{aligned}
$$

Proof Using Cauchy-Schwarz inequality, we get

$$
\begin{aligned}
& \int_{\Omega}\left(\int_{0}^{+\infty} h(s)(\Delta u(t)-\Delta u(t-s)) d s\right)^{2} d x \\
& \leq \int_{\Omega}\left(\int_{0}^{+\infty} \sqrt{h(s)} \sqrt{h(s)}(\Delta u(t)-\Delta u(t-s)) d s\right)^{2} d x \\
& \left.\leq c \int_{\Omega} \int_{0}^{+\infty} h(s)(\Delta u(t)-\Delta u(t-s)) d s\right)^{2} d x \leq c(h \circ \Delta u)(t) .
\end{aligned}
$$

Similarly, we can establish the second estimate in this lemma.

Lemma 5 Assume that $(A 1)-(A 3)$ and $(33)$ are hold. Then the functionals

$$
\begin{gathered}
\psi(t)=\int_{\Omega} u u_{t} d x \\
\chi(t)=-\int_{\Omega} u_{t} \int_{0}^{t} h(t-s)(u(t)-u(s)) d s d x
\end{gathered}
$$

satisfy, along the solutions of (17), for any $\epsilon_{0} \in(0,1)$ and $\delta>0$, the following estimates

$$
\begin{aligned}
\psi^{\prime}(t) & \leq\left\|u_{t}\right\|_{2}^{2}-\frac{\ell}{2}\|\Delta u\|_{2}^{2}-\|u\|_{2}^{2}+\int_{\Omega} u^{2} \ln |u|^{\alpha} d x \\
& +c(h o \Delta u)(t) .
\end{aligned}
$$

$$
\begin{aligned}
\chi^{\prime}(t) & \leq \delta\|\Delta u\|_{2}^{2}+\frac{c}{\delta}(h o \Delta u)(t)+\frac{c}{\delta}\left(-h^{\prime} o \Delta u\right)(t) \\
& +(\delta-(1-\ell))\left\|u_{t}\right\|_{2}^{2}+c_{\epsilon_{0}, \delta}(h o \Delta u)^{\frac{1}{1+\epsilon_{0}}}(t) .
\end{aligned}
$$


Proof Direct differentiations, using (17), we get

$$
\begin{gathered}
\psi_{1}^{\prime}=\left\|u_{t}\right\|_{2}^{2}-\|\Delta u\|_{2}^{2}-\|u\|_{2}^{2}+\int_{\Omega} \Delta u \int_{0}^{\infty} h(s) \Delta u(t-s) d s d x \\
+\int_{\Omega} u^{2} \ln |u|^{\alpha} d x .
\end{gathered}
$$

We now use Lemma 4 and Young's inequality, to obtain, for any $\mu>0$,

$$
\begin{aligned}
& \int_{\Omega} \Delta u(t)\left(\int_{0}^{\infty} h(s) \Delta u(t-s) d s\right) d x \\
& \quad \leq\left(1-\ell+\frac{\mu}{2}\right)\|\Delta u\|_{2}^{2}+\frac{1}{2 \mu}(1-\ell)(h o \Delta u)(t) .
\end{aligned}
$$

By choosing $\mu=\ell$ and combining (39) and (40), we obtain (37). To prove (38), direct differentiations, using (17), gives

$$
\begin{aligned}
& \psi_{2}^{\prime}(t)=\int_{\Omega} \Delta u \int_{0}^{\infty} h(s)(\Delta u(t)-\Delta u(t-s)) d s d x \\
& +\int_{\Omega} u \int_{0}^{\infty} h(s)(u(t)-u(t-s)) d s d x \\
& +\int_{\Omega} \int_{0}^{\infty} h(s)(\Delta u(t)-\Delta u(t-s)) d s \int_{0}^{\infty} h(s) \Delta u(s) d s d x \\
& -\int_{\Omega} u \ln |u|^{\alpha} \int_{0}^{\infty} h(s)(u(t)-u(t-s)) d s d x \\
& -\int_{\Omega} u_{t} \int_{0}^{\infty} h^{\prime}(s)(u(t)-u(t-s)) d s d x \\
& -\left(\int_{0}^{\infty} h(s) d s\right) \int_{\Omega} u_{t}^{2} d x .
\end{aligned}
$$

Similarly to 39 , we estimate the right-hand side terms of 41. So, by using Young's inequality, the first term gives, for any $\delta>0$,

$$
\begin{aligned}
& \int_{\Omega} \Delta u \int_{0}^{\infty} h(s)(\Delta u(t)-\Delta u(t-s)) d s d x \\
& \leq \frac{\delta}{4}\|\Delta u\|_{2}^{2}+\frac{c}{\delta}(h o \Delta u)(t) .
\end{aligned}
$$

Using Lemma 4. Young's and Poincaré's inequalities, the second and fifth terms lead to

$$
\begin{aligned}
& \int_{\Omega} u \int_{0}^{\infty} h(s)(u(t)-u(t-s)) d s d x \\
& \leq \frac{\delta}{4}\|\Delta u\|_{2}^{2}+\frac{c}{\delta}(h o \Delta u)(t)
\end{aligned}
$$

and

$$
\begin{aligned}
& -\int_{\Omega} u_{\infty} \int_{0}^{t} h^{\prime}(s)(u(t)-u(t-s)) d s d x \\
& \leq \delta\left\|u_{t}\right\|_{2}^{2}-\frac{c}{\delta}\left(h^{\prime} o \Delta u\right)(t) .
\end{aligned}
$$

Similarly, the third term can be estimated as follows

$$
\begin{gathered}
\int_{\Omega} \int_{0}^{\infty} h(s)(\Delta u(t)-\Delta u(t-s)) d s \int_{0}^{\infty} h(s) \Delta u(t-s) d s d x \\
\leq \frac{\delta}{4}\|\Delta u\|_{2}^{2}+c\left(1+\frac{1}{\delta}\right)(h o \Delta u)(t) .
\end{gathered}
$$

Let $\epsilon_{0} \in(0,1)$, so the following inequality holds:

$$
s|\ln s| \leq s^{2}+d_{\epsilon_{0}} s^{1-\epsilon_{0}}, \forall s>0 .
$$

Applying 46 to $u \ln |u|$, using Cauchy-Schwarz' inequality, the embedding of $H_{0}^{2}(\Omega)$ in $L^{\infty}(\Omega)$ and performing the same calculations as before, we get, for any $\delta_{1}>0$,

$$
\begin{aligned}
& \int_{\Omega} u \ln |u|^{\alpha} \int_{0}^{\infty} h(s)(u(t)-u(t-s)) d s d x \\
& \leq\left. c \delta_{1}|| \Delta u\right|_{2} ^{2}+\frac{c}{\delta_{1}} \int_{\Omega}\left|\int_{0}^{\infty} h(s)(u(t)-u(t-s)) d s\right|^{2} d x \\
& +c_{\epsilon_{0}, \delta_{1}} \int_{\Omega}\left|\int_{0}^{\infty} h(s)(u(t)-u(t-s)) d s\right|^{\frac{2}{1+\epsilon_{0}}} d x,
\end{aligned}
$$

then, putting $\frac{\delta}{4}=c \delta_{1}$ and using Holder's inequality and Lemma 4, we find

$$
\begin{aligned}
& \int_{\Omega} u \ln |u|^{\alpha} \int_{0}^{\infty} h(s)(u(t)-u(t-s)) d s d x \\
& \leq \frac{\delta}{4}\|\Delta u\|_{2}^{2}+\frac{c}{\delta}(h o \Delta u)(t)+c_{\epsilon_{0}, \delta}(h o \Delta u)^{\frac{1}{1+\epsilon_{0}}}(t) .
\end{aligned}
$$

The above inequalities imply 38 .

Lemma 6 Assume that $(A 1)-(A 3)$ and 33 hold and let $\varepsilon_{0} \in(0,1)$. Assume that

$$
0<E(0)<\frac{e \ell \pi}{4 c_{p}}
$$

Then, for $\alpha$ small enough, there exist positive constants $\varepsilon$ and $N$ such that the functional

$$
L:=E(t)+\varepsilon_{1} \psi(t)+\varepsilon_{2} \chi(t)
$$

satisfies

$$
L \sim E
$$

and, for any $t \geq 0$, there exists a positive constant $m$ such that

$$
L^{\prime}(t) \leq-m E(t)+c(h \circ \Delta u)(t)+c_{\epsilon_{0}}(h \circ \Delta u)^{\frac{1}{1+\epsilon_{0}}}(t) .
$$

Proof For the proof of (49), we see that, using similar calculations as before,

$$
\begin{aligned}
& |L(t)-E(t)|=\left|\varepsilon_{1} \psi(t)+\varepsilon_{2} \chi(t)\right| \\
& \leq c\left(\varepsilon_{1}+\varepsilon_{2}\right)\left(\left\|u_{t}\right\|_{2}^{2}+\|\Delta u\|_{2}^{2}+(h o \Delta u)(t)\right),
\end{aligned}
$$

therefore, from (34) and 32 , we obtain

$|L(t)-E(t)| \leq c\left(\varepsilon_{1}+\varepsilon_{2}\right)\left(\frac{1}{2}\left\|u_{t}\right\|_{2}^{2}+J(t)\right)=c\left(\varepsilon_{1}+\varepsilon_{2}\right) E(t)$,

then

$\left(1-c\left(\varepsilon_{1}+\varepsilon_{2}\right)\right) E(t) \leq L(t) \leq\left(1+c\left(\varepsilon_{1}+\varepsilon_{2}\right)\right) E(t)$.

Hence, for $\varepsilon_{1}, \varepsilon_{2}>0$ satisfying

$$
1-c\left(\varepsilon_{1}+\varepsilon_{2}\right)>0,
$$


the equivalence 49) holds. To prove (50), we let $\int_{0}^{+\infty} h(s) d s=: h_{0}$ and use (25), (37), (38) and the definition of $E(t)$, therefor for any $t \geq 0$ and $m>0$ we have

$$
\begin{aligned}
& L^{\prime}(t) \leq-m E(t)-\left(\varepsilon_{2}\left(h_{0}-\delta\right)-\varepsilon_{1}-\frac{m}{2}\right)\left\|u_{t}\right\|_{2}^{2} \\
& -\left(\frac{\ell}{2} \varepsilon_{1}-\varepsilon_{2} \delta-\frac{m}{2}\right)\|\Delta u\|_{2}^{2}-\left(\varepsilon_{1}-\frac{(\alpha+2) m}{4}\right)\|u\|_{2}^{2} \\
& +\left(\alpha \varepsilon_{1}-\alpha \frac{m}{2}\right) \int_{\Omega} u^{2} \ln |u| d x+\left(c \varepsilon_{1}+\varepsilon_{2} \frac{c}{\delta}+\frac{m}{2}\right)(h o \Delta u)(t) \\
& +\left(\frac{1}{2}-\frac{c \varepsilon_{2}}{\delta}\right)\left(h^{\prime} o \Delta u\right)(t)+\varepsilon_{2} c_{\epsilon_{0}, \delta}(h o \Delta u)^{\frac{1}{1+\epsilon_{0}}}(t) .
\end{aligned}
$$

Using the Logarithmic Sobolev inequality, for $0<m<$ $2 \varepsilon_{1}$, we get

$$
\begin{aligned}
& L^{\prime}(t) \leq-m E(t)-\left(\varepsilon_{2}\left(h_{0}-\delta\right)-\varepsilon_{1}-\frac{m}{2}\right)\left\|u_{t}\right\|_{2}^{2} \\
& -\left(\frac{\ell}{2} \varepsilon_{1}-\varepsilon_{2} \delta-\frac{m}{2}-\alpha\left(\varepsilon_{1}-\frac{m}{2}\right) \frac{c_{p} a^{2}}{2 \pi}\right)\|\Delta u\|_{2}^{2} \\
& -\left(\varepsilon_{1}-\frac{m(\alpha+2)}{4}+\alpha\left(\varepsilon_{1}-\frac{m}{2}\right)(1+\ln a)\right)\|u\|_{2}^{2} \ln \|u\|_{2}^{2} \\
& -\left(\alpha\left(\frac{m}{4}-\frac{\varepsilon_{1}}{2}\right)\right)\|u\|_{2}^{2}+\left(c \varepsilon_{1}+\varepsilon_{2} \frac{c}{\delta}+\frac{m}{2}\right)(h o \Delta u)(t) \\
& +\left(\frac{1}{2}-\frac{c \varepsilon_{2}}{\delta}\right)\left(h^{\prime} o \Delta u\right)(t)+\varepsilon_{2} c_{\epsilon_{0}, \delta}(h o \Delta u)^{\frac{1}{1+\epsilon_{0}}}(t) .
\end{aligned}
$$

At this point we choose $\delta$ so small that

$$
h_{0}-\delta>\frac{1}{2} h_{0} \text { and } \delta<\frac{\ell h_{0}}{16} .
$$

Whence $\delta$ is fixed, the choice of any two positive constants $\varepsilon_{1}$ and $\varepsilon_{2}$ satisfying

$$
\frac{h_{0}}{4} \varepsilon_{2}<\varepsilon_{1}<\frac{h_{0}}{2} \varepsilon_{2}
$$

will make

$$
k_{1}:=\varepsilon_{2}\left(h_{0}-\delta\right)-\varepsilon_{1}>0 \quad \text { and } \quad k_{2}:=\frac{\ell}{2} \varepsilon_{1}-\varepsilon_{2} \delta>0 .
$$

Then, we choose $\varepsilon_{1}$ and $\varepsilon_{2}$ so small so that (51) and (54) remain valid and, further,

$$
\frac{1}{2}-\frac{c \varepsilon_{2}}{\delta}>0
$$

Consequently, we get 49 and

$$
\begin{aligned}
& L(t) \leq-m E(t)-\left(k_{1}-\frac{m}{2}\right)\left\|u_{t}\right\|_{2}^{2} \\
& -\left(k_{2}-\frac{m}{2}-\alpha\left(\varepsilon_{1}-\frac{m}{2}\right) \frac{c_{p} a^{2}}{2 \pi}\right)\|\Delta u\|_{2}^{2} \\
& -\left(\varepsilon_{1}-\frac{m(\alpha+2)}{4}+\alpha\left(\varepsilon_{1}-\frac{m}{2}\right)(1+\ln a)\right)\|u\|_{2}^{2} \ln \|u\|_{2}^{2} \\
& -\left(\alpha\left(\frac{m}{4}-\frac{\varepsilon_{1}}{2}\right)\right)\|u\|_{2}^{2}+c(h o \Delta u)(t)+c_{\epsilon_{0}, \delta}(h o \Delta u)^{\frac{1}{1+\epsilon_{0}}}(t) .
\end{aligned}
$$

By choosing a satisfying (28) and $\alpha$ so small so that

$\alpha_{1}=k_{1}-\frac{m}{2}>0, \alpha_{2}=k_{2}-\frac{m}{2}-\alpha\left(\varepsilon_{1}-\frac{m}{2}\right) \frac{c_{p} a^{2}}{2 \pi}>0$ and

$$
\begin{aligned}
\alpha_{3} & =\varepsilon_{1}-\frac{m(\alpha+2)}{4}+\alpha\left(\varepsilon_{1}-\frac{m}{2}\right)(1+\ln a) \\
& +\alpha\left(\frac{m}{4}-\frac{\varepsilon_{1}}{2}\right) \ln \|u\|_{2}^{2}>0 .
\end{aligned}
$$

Therefore, we arrive at the desired result 50.

Remark 5 Recalling 24, 25, 30 and (34), we have $E(0) \geq E(t)=J(t)+\frac{1}{\rho+2}\left\|u_{t}\right\|_{\rho+2}^{\rho+2} \geq J(t) \geq \frac{1}{3}(h \circ \Delta u)(t)$,

which gives

$$
(h \circ \Delta u)(t) \leq 3 E(0) .
$$

Using (56), we obtain the following

$$
\begin{gathered}
(h \circ \Delta u)(t)=(h \circ \Delta u)^{\frac{\epsilon_{0}}{1+\epsilon_{0}}}(t)(h \circ \Delta u)^{\frac{1}{1+\epsilon_{0}}}(t) \\
\leq c(h \circ \Delta u)^{\frac{1}{1+\epsilon_{0}}}(t)
\end{gathered}
$$

Lemma 7 If $(A 1)-(A 2)$ are satisfied, then we have, for all $t>0$, the following estimate

$$
\int_{0}^{t} h(s)\|\Delta u(t)-\Delta(t-s)\|_{2}^{2} d s \leq \frac{t}{q} H^{-1}\left(\frac{q \mu(t)}{t \xi(t)}\right)
$$

where $q$ small enough, $H$ is defined in Remark (2) and

$$
\mu(t):=-\int_{0}^{t} h^{\prime}(s) \| \Delta u(t)-\left.\Delta(t-s)\right|_{2} ^{2} d s \leq-c E^{\prime}(t)
$$

Proof To establish 58, we introduce the following functional

$$
\lambda(t):=\frac{q}{t} \int_{0}^{t}\|\Delta u(t)-\Delta(t-s)\|_{2}^{2} d s .
$$

Then, using the fact that $E$ is nonincreasing and (24) to get

$$
\begin{aligned}
\lambda(t) \leq & \frac{2 q}{t}\left(\int_{0}^{t}\|\Delta u(t)\|_{2}^{2}+\int_{0}^{t}\|\Delta(t-s)\|_{2}^{2} d s\right) . \\
& \leq \frac{4 q}{\ell t}\left(\int_{0}^{t}(E(t)+E(t-s)) d s\right) \\
& \leq \frac{8 q}{\ell t} \int_{0}^{t} E(s) d s \\
& \leq \frac{8 q}{\ell t} \int_{0}^{t} E(0) d s \\
& <+\infty
\end{aligned}
$$

Thus, $q$ can be chosen so small so that, for all $t>0$,

$$
\lambda(t)<1
$$

Without loss of the generality, for all $t>0$, we assume that $\mu(t)>0$, otherwise we get an exponential decay from 
(50). The use of Jensen's inequality and using (59), (3) and 62 gives

$$
\begin{aligned}
& \mu(t)=\frac{1}{q \lambda(t)} \int_{0}^{t} \lambda(t)\left(-h^{\prime}(s)\right) \int_{\Omega} q|\Delta u(t)-\Delta(t-s)|^{2} d x d s \\
& \geq \frac{1}{q \lambda(t)} \int_{0}^{t} \lambda(t) \xi(s) H(h(s)) \int_{\Omega} q|\Delta u(t)-\Delta(t-s)|^{2} d x d s \\
& \geq \frac{\xi(t)}{q \lambda(t)} \int_{0}^{t} H(\lambda(t) h(s)) \int_{\Omega} q|\Delta u(t)-\Delta(t-s)|^{2} d x d s \\
& \geq \frac{t \xi(t)}{q} H\left(\frac{q}{t} \int_{0}^{t} h(s) \int_{\Omega}|\Delta u(t)-\Delta(t-s)|^{2} d x d s\right) \\
& =\frac{t \xi(t)}{q} \bar{H}\left(\frac{q}{t} \int_{0}^{t} h(s) \int_{\Omega}|\Delta u(t)-\Delta u(t-s)|^{2} d x d s\right),
\end{aligned}
$$

hence (58) is established.

\section{ENERGY DECAY}

In this section, we state and prove our main general decay result. Our decay result is in the following theorem.

Theorem 2 Let $\left(u_{0}, u_{1}\right) \in H_{0}^{2}(\Omega) \times L^{2}(\Omega)$. Assume that $(A 1)-(A 3)$ and $(33)$ hold. Then, there exist positive constants $C_{1}, C_{2}$ such that the solution of (17) satisfies, for all $t \geq t_{1}$,

$$
E(t) \leq \frac{C_{1}}{\gamma(t)} \Phi_{2}^{-1}\left(\frac{C_{2} \gamma(t)}{\int_{t_{1}}^{t} \xi(s) d s}\right) .
$$

where the functions $\gamma(s), \Phi_{2}(s)$ are functions will be defined in the proof.

Proof Combining (50), (57) and (58), then for any $t \geq$ $t_{0}$, we get

$$
L^{\prime}(t) \leq-m E(t)+c\left(\frac{t}{q}\right)^{\frac{1}{1+\epsilon_{0}}}\left(H^{-1}\left(\frac{q \mu(t)}{t \xi(t)}\right)\right)^{\frac{1}{1+\epsilon_{0}}}(t) .
$$

Combining the strictly increasing property of $H$ and the fact $\frac{1}{t}<1$ whenever $t>1$, we obtain

$$
H^{-1}\left(\frac{q \mu(t)}{t \xi(t)}\right) \leq H^{-1}\left(\frac{q \mu(t)}{(t)^{\frac{1}{1+\epsilon_{0}}} \xi(t)}\right),
$$

and, then, 65 becomes, for $t_{1}=\max \left\{t_{0}, 1\right\}$ and for any $t \geq t_{1}$

$$
L^{\prime}(t) \leq-m E(t)+c_{\epsilon_{0}} \frac{(t)^{\frac{1}{1+\epsilon_{0}}}}{q}\left(H^{-1}\left(\frac{q \mu(t)}{(t)^{\frac{1}{1+\epsilon_{0}}} \xi(t)}\right)\right)^{\frac{1}{1+\epsilon_{0}}} .
$$

For simplicity, we let $\gamma(t)=: \frac{q}{(t)^{\frac{1}{1+\epsilon_{0}}}}$. Then, 67) becomes

$$
L^{\prime}(t) \leq-m E(t)+\frac{c_{\epsilon_{0}}}{\gamma(t)}\left(H^{-1}\left(\frac{\gamma(t) \mu(t)}{\xi(t)}\right)\right)^{\frac{1}{1+\epsilon_{0}}} .
$$

Further, letting $\chi(t)=\left(\frac{\gamma(t) \mu(t)}{\xi(t)}\right)$ and

$$
\Phi^{-1}(\chi(t))=:\left(H^{-1}(\chi(t))\right)^{\frac{1}{1+\epsilon_{0}}} .
$$

Then, 68 reduces to

$$
L^{\prime}(t) \leq-m E(t)+\frac{c_{\epsilon_{0}}}{\gamma(t)} \Phi^{-1}(\chi(t)), \forall t \geq t_{1} .
$$

Now, for $\varepsilon<r$ and using the fact that $E^{\prime} \leq 0, \Phi^{\prime}>$ $0, \Phi^{\prime \prime}>0$ on $(0, r]$, we find that the functional $L_{1}$, defined by

$$
L_{1}(t):=\Phi^{\prime}\left(\varepsilon \cdot \frac{\gamma(t) E(t)}{E(0)}\right) L(t), \quad \forall t \geq t_{1},
$$

satisfies, for some $\alpha_{1}, \alpha_{2}>0$,

$$
\alpha_{1} L_{1}(t) \leq E(t) \leq \alpha_{2} L_{1}(t) .
$$

Using the fact $(\gamma E)^{\prime}(t) \leq 0$ and $\Phi^{\prime}>0$, therefore, combining (70) with (69), we arrive at

$$
\begin{aligned}
L_{1}^{\prime}(t) & \leq-m E(t) \Phi^{\prime}\left(\varepsilon \cdot \frac{\gamma(t) E(t)}{E(0)}\right) \\
& +\frac{c_{\epsilon_{0}}}{\gamma(t)} \Phi^{\prime}\left(\varepsilon \cdot \frac{\gamma(t) E(t)}{E(0)}\right) \Phi^{-1}(\chi(t)) .
\end{aligned}
$$

Let $\Phi^{*}$ be the convex conjugate of $\Phi$ in the sense of Young (see [37]), then

$$
\Phi^{*}(s)=s\left(\Phi^{\prime}\right)^{-1}(s)-\Phi\left[\left(\Phi^{\prime}\right)^{-1}(s)\right], \quad \text { if } s \in\left(0, \Phi^{\prime}(r)\right]
$$

and $\Phi^{*}$ satisfies the following generalized Young inequality

$$
A B \leq \Phi^{*}(A)+\Phi(B), \quad \text { if } A \in\left(0, \Phi^{\prime}(r)\right], B \in(0, r] .
$$

Using (73) and the generalized Young inequality (74) on the last term in 72 with $A=\Phi^{\prime}\left(\varepsilon \cdot \frac{\gamma(t) E(t)}{E(0)}\right)$ and $B=$ $\Phi^{-1}(\chi(t))$, we get

$$
\begin{aligned}
L_{1}^{\prime}(t) & \leq-m E(t) \Phi^{\prime}\left(\varepsilon \cdot \frac{\gamma(t) E(t)}{E(0)}\right)+\frac{c_{\epsilon_{0}}}{\gamma(t)} \Phi^{*}\left(\Phi^{\prime}\left(\varepsilon \cdot \frac{\gamma(t) E(t)}{E(0)}\right)\right) \\
& +\frac{c_{\epsilon_{0}}}{\gamma(t)} \chi(t) \\
& \leq-m E(t) \Phi^{\prime}\left(\varepsilon \cdot \frac{\gamma(t) E(t)}{E(0)}\right)+c_{\epsilon_{0}} \varepsilon \frac{E(t)}{E(0)} \Phi^{\prime}\left(\varepsilon \cdot \frac{\gamma(t) E(t)}{E(0)}\right) \\
& +\frac{c_{\epsilon_{0}}}{\gamma(t)} \chi(t) .
\end{aligned}
$$

Multiplying both sides of 75 by $\xi(t)$ and using the fact that $\frac{\xi(t)}{\gamma(t)} \chi(t) \leq-c E^{\prime}(t)$, we obtain

$$
\begin{aligned}
& \xi(t) L_{1}^{\prime}(t) \leq-m \xi(t) E(t) \Phi^{\prime}\left(\varepsilon \cdot \frac{\gamma(t) E(t)}{E(0)}\right) \\
& +c_{\epsilon_{0} \varepsilon} \xi(t) \frac{E(t)}{E(0)} \Phi^{\prime}\left(\varepsilon \cdot \frac{\gamma(t) E(t)}{E(0)}\right) \\
& -c_{\epsilon_{0}} E^{\prime}(t) .
\end{aligned}
$$

Using the non-increasing property of $\xi(t)$, we obtain, for all $t \geq t_{1}$,

$$
\begin{aligned}
& \left(\xi L_{1}+c_{\epsilon_{0}} E\right)^{\prime}(t) \leq-m \xi(t) E(t) \Phi^{\prime}\left(\varepsilon \cdot \frac{\gamma(t) E(t)}{E(0)}\right) \\
& +c_{\epsilon_{0}} \varepsilon \xi(t) \frac{E(t)}{E(0)} \Phi^{\prime}\left(\varepsilon \cdot \frac{\gamma(t) E(t)}{E(0)}\right) .
\end{aligned}
$$


Therefore, by setting $L_{2}:=\xi L_{1}+c_{\epsilon_{0}} E \sim E$, we get

$$
\begin{aligned}
& L_{2}^{\prime}(t) \leq-m \xi(t) E(t) \Phi^{\prime}\left(\varepsilon \cdot \frac{\gamma(t) E(t)}{E(0)}\right) \\
& +c_{\epsilon_{0}} \varepsilon \xi(t) \frac{E(t)}{E(0)} \Phi^{\prime}\left(\varepsilon \cdot \frac{\gamma(t) E(t)}{E(0)}\right) .
\end{aligned}
$$

This gives, for a suitable choice of $\varepsilon$ so that $k=m-$ $c_{\epsilon_{0}} \varepsilon>0$,

$$
L_{2}^{\prime}(t) \leq-k \xi(t) \frac{E(t)}{E(0)} \Phi^{\prime}\left(\varepsilon \cdot \frac{\gamma(t) E(t)}{E(0)}\right), t \geq t_{1} .
$$

Integrating 79 over $\left(t_{1}, t\right)$ gives

$\int_{t_{1}}^{t} k \xi(s) \frac{E(t)}{E(0)} \Phi^{\prime}\left(\varepsilon \cdot \frac{\gamma(s) E(s)}{E(0)}\right) \leq-\int_{t_{1}}^{t} L_{2}^{\prime}(s) d s \leq L_{2}\left(t_{1}\right)$.

Using the facts that $\Phi^{\prime}, \Phi^{\prime \prime}>0$ and the nonincreasing property of $E$, we deduce that the map $t \mapsto$ $E(t) \Phi^{\prime}\left(\varepsilon \cdot \frac{\gamma(s) E(s)}{E(0)}\right)$ is non-increasing and consequently, we have for any $t \geq s \geq t_{1}$

$$
\begin{aligned}
& k \frac{E(t)}{E(0)} \Phi^{\prime}\left(\varepsilon \cdot \frac{\gamma(t) E(t)}{E(0)}\right) \int_{t_{1}}^{t} \xi(s) d s \\
& \leq \int_{t_{1}}^{t} k \xi(s) \frac{E(t)}{E(0)} \Phi^{\prime}\left(\varepsilon \cdot \frac{\gamma(s) E(s)}{E(0)}\right) \\
& \leq L_{2}\left(t_{1}\right) .
\end{aligned}
$$

Multiplying each side of (81) by $\gamma(t)$, we have for $k_{1}>0$,

$$
k \frac{\gamma(t) E(t)}{E(0)} \Phi^{\prime}\left(\varepsilon \cdot \frac{\gamma(t) E(t)}{E(0)}\right) \int_{t_{0}}^{t} \xi(s) d s \leq k_{1} \gamma(t) .
$$

Next, we set $\Phi_{2}(s)=s \Phi^{\prime}(\varepsilon s)$ which is strictly increasing, and then we obtain for any $t \geq t_{1}$ and some constant $k_{2}>0$,

$$
\Phi_{2}\left(\varepsilon \cdot \frac{\gamma(t) E(t)}{E(0)}\right) \int_{t_{1}}^{t} \xi(s) d s \leq k_{2} \gamma(t)
$$

This gives, for any $t \geq t_{0}$ and some constant $k_{3}>0$,

$$
E(t) \leq \frac{k_{3}}{\gamma(t)} \Phi_{2}^{-1}\left(\frac{k_{2} \gamma(t)}{\int_{t_{1}}^{t} \xi(s) d s}\right)
$$

This finishes the proof.

Example 1 The following example illustrates our results:

Let $h(t)=\frac{a}{(1+t)^{q}}$, where $q>1+\epsilon_{0}$ and $a$ is chosen so that hypothesis $\left(A_{1}\right)$ remains valid. Then

$$
h^{\prime}(t)=-b H(h(t)), \quad \text { with } \quad H(s)=s^{\frac{q+1}{q}},
$$

where $b$ is a fixed constant. Since $\Phi(s)=s^{\frac{\left(\epsilon_{0}+1\right)(q+1)}{q}}$. Then, the estimate 64 gives, $\forall t \geq t_{1}$

$$
E(t) \leq c t^{\frac{-\left(q-1-\epsilon_{0}\right)}{\left(1+\epsilon_{0}\right)^{2}(q+1)}} .
$$

\section{CONCLUSions AND FUtURE WORKS}

We succeed to prove the decay results known for the case of finite history to the case of infinite history where the relaxation function satisfies a wider class of relaxation functions. Note that our decay result (64) generalizes the once of [22], 24], [38, 39], [40, 41] and 25]. For the future works, we plan to find a class of relaxation functions for which we obtain an optimal decay.

\section{ACKNOWLEDGMENT}

The author thanks King Fahd University of Petroleum and Minerals (KFUPM) for its continuous support and an anonymous referee for her/his careful reading and valuables remarks. This work is funded by KFUPM under Project \# SB181039.

\section{REFERENCES}

[1] C. M. Dafermos, "Asymptotic stability in viscoelasticity," Archive for rational mechanics and analysis, vol. 37, no. 4, pp. 297-308, 1970.

[2] J. A. Appleby, M. Fabrizio, B. Lazzari, and D. W. Reynolds, "On exponential asymptotic stability in linear viscoelasticity," Mathematical Models and Methods in Applied Sciences, vol. 16, no. 10, pp. 1677-1694, 2006.

[3] V. Pata, "Stability and exponential stability in linear viscoelasticity," Milan journal of mathematics, vol. 77 , no. 1, p. 333, 2009.

[4] A. Guesmia, "Asymptotic stability of abstract dissipative systems with infinite memory," Journal of Mathematical Analysis and Applications, vol. 382, no. 2, pp. 748-760, 2011.

[5] A. M. Al-Mahdi and M. M. Al-Gharabli, "New general decay results in an infinite memory viscoelastic problem with nonlinear damping," Boundary Value Problems, vol. 2019, no. 1, p. 140, 2019.

[6] M. I. Mustafa, "Energy decay in a quasilinear system with finite and infinite memories," in Mathematical Methods in Engineering, pp. 235-256, Springer, 2019.

[7] A. M. Al-Mahdi, "Stability result of a viscoelastic plate equation with past history and a logarithmic nonlinearity," Boundary Value Problems, vol. 2020, no. 1, pp. 1-20, 2020.

[8] A. M. Al-Mahdi, "General stability result for a viscoelastic plate equation with past history and general kernel," Journal of Mathematical Analysis and Applications, p. 124216, 2020.

[9] "A numerical analysis of viscoelastic flow between porous moving walls", Wseas Transactions on Applied and Theoretical Mechanics, 14, 212-221, 2019.,"

[10] F. Alabau-Boussouira and P. Cannarsa, "A general method for proving sharp energy decay rates for memory-dissipative evolution equations," Comptes Rendus Mathématique, vol. 347, no. 15-16, pp. 867872, 2009.

[11] M. M. Cavalcanti, A. D. Cavalcanti, I. Lasiecka, and $\mathrm{X}$. Wang, "Existence and sharp decay rate estimates 
for a von karman system with long memory," Nonlinear Analysis: Real World Applications, vol. 22, pp. 289-306, 2015.

[12] B. Feng and T. A. Apalara, "Optimal decay for a porous elasticity system with memory," Journal of Mathematical Analysis and Applications, vol. 470, no. 2, pp. 1108-1128, 2019.

[13] M. M. Al-Gharabli, A. M. Al-Mahdi, and S. A. Messaoudi, "General and optimal decay result for a viscoelastic problem with nonlinear boundary feedback," Journal of Dynamical and Control Systems, vol. 25, no. 4, pp. 551-572, 2019.

[14] X.-G. Yang, J. Zhang, and S. Wang, "Stability and dynamics of a weak viscoelastic system with memory and nonlinear time-varying delay," Discrete $\&$ Continuous Dynamical Systems-A, vol. 40, no. 3, p. $1493,2020$.

[15] M. Conti and V. Pata, "General decay properties of abstract linear viscoelasticity," Zeitschrift für angewandte Mathematik und Physik, vol. 71, no. 1, p. 6, 2020.

[16] A. M. Al-Mahdi, M. M. Al-Gharabli, and M. Kafini, "A new general decay result for abstract evolution equation with time-dependent nonlinear dissipation," ANNALI DELL'UNIVERSITA'DI FER$R A R A$, vol. 65, no. 2, pp. 201-230, 2019.

[17] A. M. Al-Mahdi, "Optimal decay result for kirchhoff plate equations with nonlinear damping and very general type of relaxation functions," Boundary Value Problems, vol. 2019, no. 1, p. 82, 2019.

[18] S. E. Mukiawa, "Decay result for a delay viscoelastic plate equation," Bulletin of the Brazilian Mathematical Society, New Series, pp. 1-24, 2019.

[19] "On static hierarchical two-dimensional models of thermoelastic piezoelectric plates with variable thickness", Wseas Transactions on Applied and Theoretical Mechanics, 13, 2224-3429, 2018.,"

[20] "Increasing accuracy of discrete-time computation of linear rheological and viscoelastic material functions", Wseas Transactions on Applied and Theoretical Mechanics, 14, 15-20, 2019.,"

[21] I. Bialynicki-Birula and J. Mycielski, "Wave equations with logarithmic nonlinearities," Bull. Acad. Polon. Sci. Cl, vol. 3, no. 23, p. 461, 1975.

[22] P. Gorka, "Logarithmic klein-gordon equation," Acta Phys. Polon., vol. 40, pp. 59-66, 2009.

[23] K. Bartkowski and P. Górka, "One-dimensional klein-gordon equation with logarithmic nonlinearities," Journal of Physics A: Mathematical and Theoretical, vol. 41, no. 35, p. 355201, 2008.

[24] T. Hiramatsu, M. Kawasaki, and F. Takahashi, "Numerical study of q-ball formation in gravity mediation," Journal of Cosmology and Astroparticle Physics, vol. 2010, no. 06, p. 008, 2010.

[25] M. M. Al-Gharabli, A. Guesmia, and S. A. Messaoudi, "Existence and a general decay results for a viscoelastic plate equation with a logarithmic nonlinearity.," Communications on Pure \& Applied Analysis, vol. 18, no. 1, 2019.
[26] M. M. Al-Gharabli, A. Guesmia, and S. A. Messaoudi, "Well-posedness and asymptotic stability results for a viscoelastic plate equation with a logarithmic nonlinearity," Applicable Analysis, pp. 1-25, 2018.

[27] I. Bialynicki-Birula and J. Mycielski, "Nonlinear wave mechanics," Annals of Physics, vol. 100, no. 12, pp. 62-93, 1976.

[28] T. Cazenave and A. Haraux, "Équations d'évolution avec non linéarité logarithmique," in Annales de la Faculté des sciences de Toulouse: Mathématiques, vol. 2, pp. 21-51, 1980.

[29] M. M. Al-Gharabli, "New general decay results for a viscoelastic plate equation with a logarithmic nonlinearity," Boundary Value Problems, vol. 2019, no. 1, pp. 1-21, 2019.

[30] X. Han, "Global existence of weak solutions for a logarithmic wave equation arising from q-ball dynamics," Bulletin of the Korean Mathematical Society, vol. 50, no. 1, pp. 275-283, 2013.

[31] W. Lian and R. Xu, "Global well-posedness of nonlinear wave equation with weak and strong damping terms and logarithmic source term," Advances in Nonlinear Analysis, vol. 9, no. 1, pp. 613-632, 2019.

[32] A. Peyravi, "General stability and exponential growth for a class of semi-linear wave equations with logarithmic source and memory terms," $A p$ plied Mathematics \& Optimization, pp. 1-17, 2018.

[33] R. Xu, W. Lian, X. Kong, and Y. Yang, "Fourth order wave equation with nonlinear strain and logarithmic nonlinearity," Applied Numerical Mathematics, vol. 141, pp. 185-205, 2019.

[34] X. Wang, Y. Chen, Y. Yang, J. Li, and R. Xu, "Kirchhoff-type system with linear weak damping and logarithmic nonlinearities," Nonlinear Analysis, vol. 188, pp. 475-499, 2019.

[35] L. Gross, "Logarithmic sobolev inequalities," American Journal of Mathematics, vol. 97, no. 4, pp. 1061-1083, 1975.

[36] H. Chen, P. Luo, and G. Liu, "Global solution and blow-up of a semilinear heat equation with logarithmic nonlinearity," Journal of Mathematical Analysis and Applications, vol. 422, no. 1, pp. 84-98, 2015.

[37] V. I. Arnol'd, Mathematical methods of classical mechanics, vol. 60. Springer Science \& Business Media, 2013.

[38] S. A. Messaoudi and W. Al-Khulaifi, "General and optimal decay for a quasilinear viscoelastic equation," Applied Mathematics Letters, vol. 66, pp. 16$22,2017$.

[39] M. I. Mustafa, "Optimal decay rates for the viscoelastic wave equation," Mathematical Methods in the Applied Sciences, vol. 41, no. 1, pp. 192-204, 2018.

[40] M. M. Cavalcanti, V. N. D. Cavalcanti, I. Lasiecka, and C. M. Webler, "Intrinsic decay rates for the energy of a nonlinear viscoelastic equation modeling the vibrations of thin rods with variable den- 
sity," Advances in Nonlinear Analysis, vol. 6, no. 2, pp. 121-145, 2017.

[41] M. M. Al-Gharabli, A. Guesmia, and S. A. Messaoudi, "Well-posedness and asymptotic stability results for a viscoelastic plate equation with a logarithmic nonlinearity," Applicable Analysis, pp. 1-25, 2018.

\section{Creative Commons Attribution License 4.0} (Attribution 4.0 International, CC BY 4.0)

This article is published under the terms of the Creative Commons Attribution License 4.0

https://creativecommons.org/licenses/by/4.0/deed.en_US 